\title{
Enormous Initial Cerebellar Metastasis Revealing Papillary Carcinoma of the Thyroid: Rare Case
}

\author{
Said Anajar*, Mohammed Moutaa Tatari, Jawad Hassnaoui, Redallah Abada, Sami Rouadi, Mohammed Roubal, \\ Mohammed Mahtar
}

ENT Department, Face and Neck Surgery, Hospital August, 20’1953, University Hospital Centre IBN ROCHD, Casablanca, Morrocco *Corresponding author: anajar.said.med@gmail.com

\begin{abstract}
Introduction: Papillary carcinoma is the most frequent differentiated malignant thyroid neoplasm. Metastasis occurs frequently in regional lymph nodes and remote metastasis are late and rare, only 16 cases of initial cerebellar metastasis have been reported in the literature. Results: We report a rare case of cerebellar metastasis revealing papillary thyroid carcinoma in a 32-year-old patient, with a review of the literature on clinical features, radiological aspect, and treatment options. Discussion Conclusion: Cerebral metastasis of thyroid cancer are rare and the initial cerebellar metastasis revealing papillary carcinoma are exceptional, and due to their rarities and the relative lack of data on their management there is no treatment algorithm clearly defined.
\end{abstract}

Keywords: cerebellar metastasis, papillary thyroid carcinoma, thyroid cance, case report

Cite This Article: Said Anajar, Mohammed Moutaa Tatari, Jawad Hassnaoui, Redallah Abada, Sami Rouadi, Mohammed Roubal, and Mohammed Mahtar, "Enormous Initial Cerebellar Metastasis Revealing Papillary Carcinoma of the Thyroid: Rare Case.” American Journal of Medical Case Reports, vol. 5, no. 3 (2017): 62-64. doi: 10.12691/ajmcr-5-3-5.

\section{Introduction}

Papillary carcinoma of the thyroid is the most common cancer of the thyroid accounting for about $80 \%$ to $90 \%$ of thyroid cancers [1]. The most common sites of revealing metastases are lung and bone [1]. Cerebral metastases are very rare [2]. The presence of distant metastases is an element of poor prognosis associated with a decrease in survival rates [3]. Initial cerebellar metastases are extremely rare with about 16 cases reported in the literature [4]. We present an exceptional case of an enormous initial cerebellar metastasis revealing thyroid cancer.

\section{Case Report}

A 32 years old patient present in the emergency room with progressive vertigo, balance disorder, persistent headache and vomiting evolving for 1 month, a cerebral scan showed an enormous vascularized and enhancing mass measuring $40-57-46 \mathrm{~mm}$ in the posterior cerebral fossa, with oedema around the lesion (Figure 1). The patient was addressed to the neurosurgery department. A Computerized Tomography-guided biopsy was performed. The histology revealing a papillary carcinoma compatible with a thyroid origin. Ultrasound and cervico-thoracic CT- scan (Figure 2) objectified a slightly enlarged thyroid-sized with two nodules classifying TI-RADS 4B (Thyroid Imaging-Reporting and Database System.). Scintigraphy of whole body performed four Days after oral administration of $3700 \mathrm{MBq}$ of iodine-131 showed fixation at the brain and thyroid levels, no other focal point was detected, TSH and thyroglobulin were normal. Total thyroidectomy was decided (Figure 3). Histology was compatible with papillary carcinoma of the thyroid. 15 days later, large resection of the cerebellar tumor was done. Then he was transferred to intensive care unit. He died 10 days after surgery.

\section{Discussion}

The most common sites of papillary thyroid carcinoma metastasis are usually in regional lymph nodes, lungs and bones, while the brain is a rare site of metastases found only in $0.15-1.3 \%$ of cases [1].

In addition, it is extremely rare for cerebellar metastasis to occur as an initial distal metastasis of papillary carcinoma of the thyroid, only 16 cases have been published to date [4].

A cerebral metastasis of thyroid cancer could be a factor of poor prognosis and median survival in these cases was 4.7 months for Misaki et al [5] and maximum of 29.2 months for Kim et al.

Patients with papillary carcinoma of the thyroid and with metastases to other organs also reduced their survival after the diagnosis of a cerebral metastasis [7].

There are no features of MRI (Magnetic resonance imaging) or other radiographic abnormalities that distinguish metastatic lesions from thyroid carcinoma of other brain tumors [7]. McWilliams et al [7] reported that cerebral metastasis of papillary carcinoma of the thyroid is relatively hypervascular. 


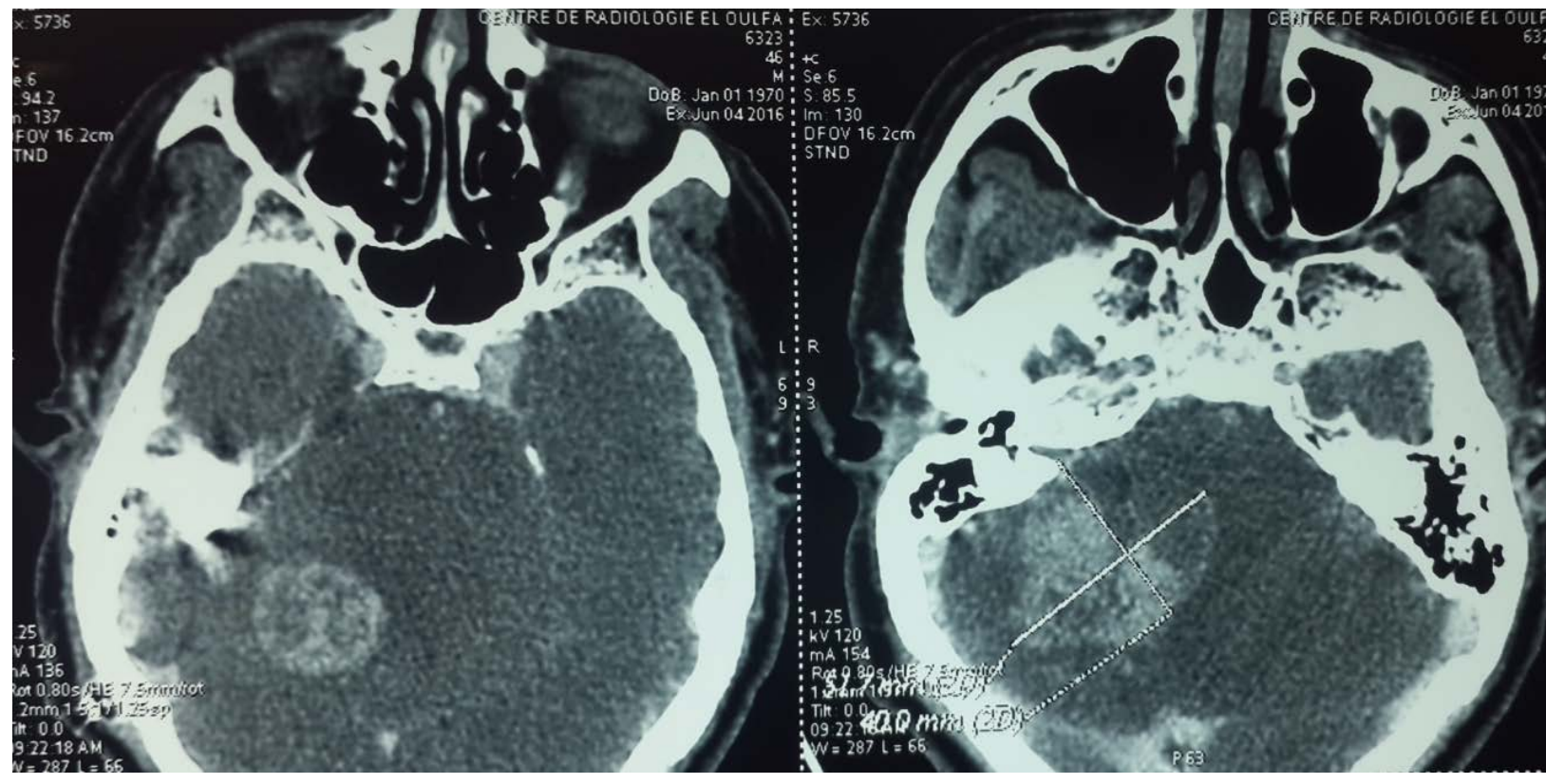

Figure 1. CEREBRAL CT SCAN SHOWING A MASS OF 40 -57- 46 MM IN THE POSTERIOR CEREBRAL FOSSA WITH OEDEMA AROUND THE LESION

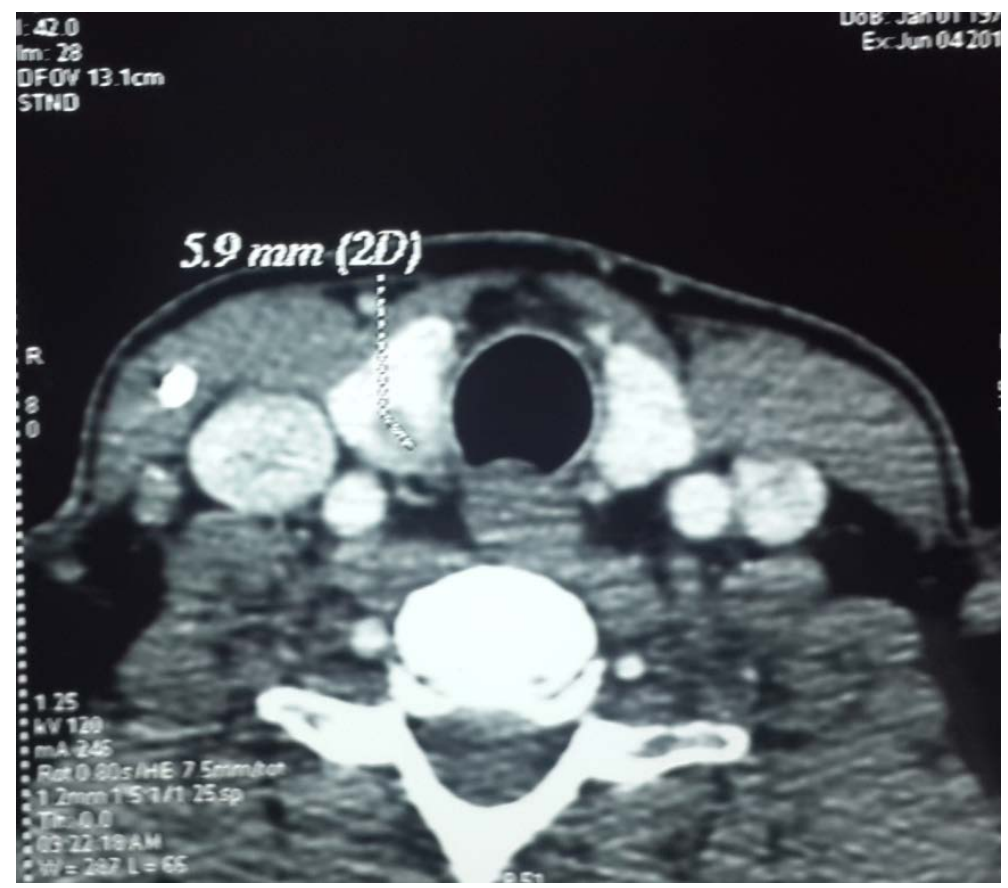

Figure 2. CERVICAL CT SCAN SHOWING A NODULAR THYROID
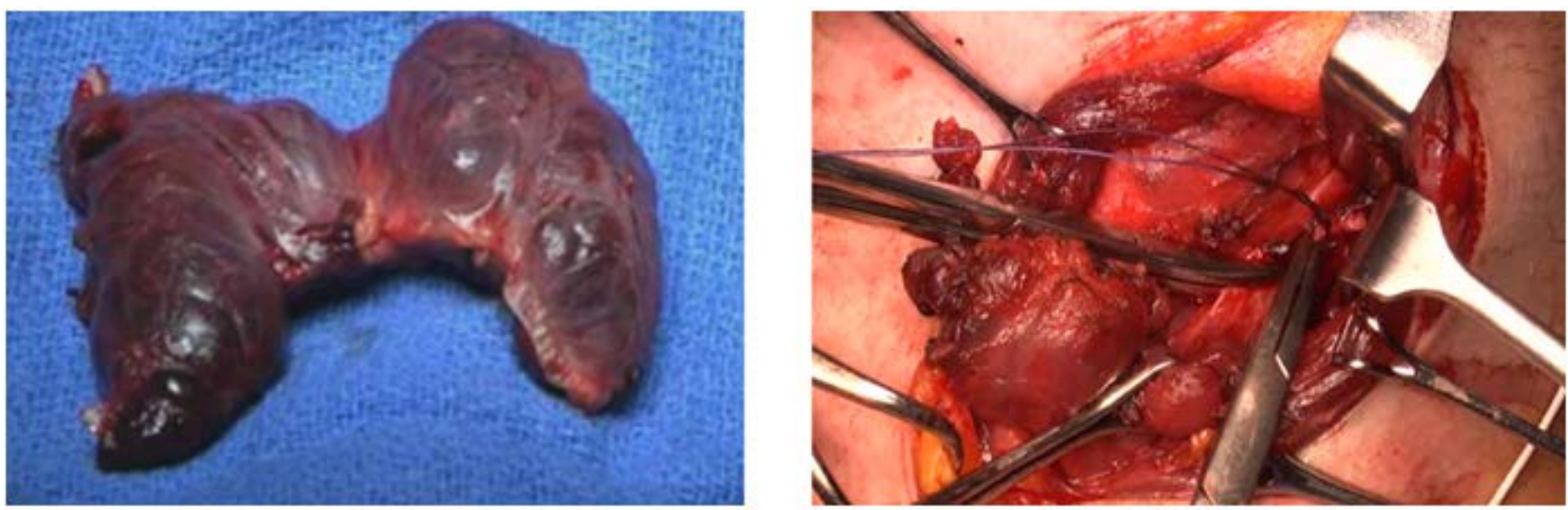

Figure 3. TOTAL THYROIDECTOMY 
There is no clearly defined protocol for the treatment of intracranial metastases of thyroid cancer, but surgery is generally considered the best choice for prolonged survival and regression of neurological symptoms [6].

Chiu et al. (1997) [8] reported a significant increase in median survival (16.7 months) for patients who underwent surgical resection of their metastases, compared to 3.4 months for those who did not benefit from surgery.

It is possible that radiotherapy is generally a candidate therapy for a single small metastasis, in our case the size was 40 -57- $46 \mathrm{~mm}$ [9].

Previous reports have reported an apparent benefit of iodo-therapy when the scintigraphy is positive. There is an excellent study that reports the survival of a 15-year-old girl with diffuse cerebral metastases after receiving treatment with high-dose age-appropriate radioactive iodine [10].

On the other hand, McWilliams et al [7] found no place for chemotherapy in patients with cerebral metastases of a thyroid carcinoma.

\section{Conclusion}

Due to the rarity of these metastases and the relative lack of data on their management, there is no clearly defined treatment algorithm for patients with intracranial metastasis. Surgical resection is considered the basic treatment of solitary intracranial lesions.

\section{Acknowledgements}

None.

\section{Conflicts of Interest}

All the authors have no personal or financial conflicts of interest regard this case report

\section{Funding}

No sources of funding to declare.

\section{Consent}

Written informed consent was obtained from the patient for publication of this case report and accompanying image.

\section{References}

[1] El Fellah S, Ksiar R, Missoum F, Ben Rais N. Métastase du tissu mou révélant un carcinome folliculaire différencié de la thyroïde. Med Nucl 2008; 32:308-10.

[2] Hamid HA, Gee KY, Muhammad R, Abd Rahman ZA, Das S. Dural metastasis mimicking meningioma: an interesting case. Acta Medica 2009; 52: 19-22.

[3] Pazaitou-Panayiotou K, Kaprara A, Chrisoulidou A, Boudina M, Georgiou E, Patakiouta F, Drimonitis A, Vainas I. Cerebellar metastasis as first metastasis from papillary thyroid carcinoma. Endocr J 2005; 52: 653-657.

[4] Tunio MA, Al Asiri M, Al-Qahtani KH, AlShakweer W Cerebellum as initial site of distant metastasis from papillary carcinoma of thyroid: review of three cases. Case Rep Neurol Med 2015: 171509

[5] Misaki T, Iwata M, Kasagi K, Konishi J. Brain metastasis from differentiated thyroid cancer in patients treated with radioiodine for bone and lung lesions. Ann Nucl Med 2000; 14: 111-114.

[6] Kim IY, Kondziolka D, Niranjan A, Flickinger JC, Lunsford LD. Gamma knife radiosurgery for metastatic brain tumors from thyroid cancer. J Neurooncol 2009 ; 93:355-359.

[7] McWilliams RR, Giannini C, Hay ID, Atkinson JL, Stafford SL, Buckner JC Management of brain metastases from thyroid carcinoma: a study of 16 pathologically confirmed cases over 25 years. Cancer 2003: 98: 356-362.

[8] Chiu AC, Delpassand ES, Sherman SI. Prognosis and treatment of brain metastases in thyroid carcinoma. J Clin Endocrinol Metab 1997; 82: 3637-3642.

[9] Muacevic A, Wowra B, Siefert A, Tonn JC, Steiger HJ, Kreth FW. Microsurgery plus whole brain irradiation versus Gamma Knife surgery alone for treatment of single metastases to the brain: a randomized controlled multicentre phase III trial. J Neurooncol 2008; 87:299-307.

[10] Vrachimis A, Schmid KW, Jurgens H, Schober O, Weckesser M, Riemann B. Cerebral metastases from thyroid carcinoma: complete remission following radioiodine treatment. Dtsch Arztebl Int 2013; 110: 861-866. 